\title{
The Development of New Data Quality Visualisation Methods in Electronic Chart Information Systems and Investigation into Associated User Response
}

Sam HARPER, United Kingdom

Seabed Data Centre, United Kingdom Hydrographic Office

David WELLS,

Hydrographic Science Research Centre, Department of Marine Science, The University of Southern Mississippi

Kandice GUNNING,

Hydrographic Science Research Centre, Department of Marine Science, The University of Southern Mississippi

Topic: D Smart data management; E Easy customer access to data and products; and G Cost-effective solutions

\section{INTRODUCTION}

The representation of geospatial data quality in a GIS environment is well researched with many different methods employed. The same level of research does not exist for the representation of data quality in ECDIS. There is concern amongst the international hydrographic community that the current methods of representing data quality in navigational products are not meeting the needs of the mariner. Instead they rely heavily on the user's ability to understand the relevance of data quality indicators such as 'survey date' and 'acquisition method', or composite quality classifications like CATZOC.

The International Hydrographic Organisation (IHO) Data Quality Working Group (DQWG) was formed to address this problem by developing a new method of representing data quality in future S-101 ENCs. So far the work of the DQWG has been centred around the creation of the S-101 Data Quality model which provides the architecture on which new visualisation methods can be built. To support this work, a questionnaire on the mariners' current perception of data quality was produced by the DQWG, and distributed by the IHO to all member states and made available to the wider marine community. The analysis of the data has lead to the development of a specification, against which a new method of data quality representation can be designed.

This paper provides a review of this work to date, and an insight into future collaborative research between the DQWG and the University of Southern Mississippi (USM).

\section{IHO DATA QUALITY WORKING GROUP QUESTIONNAIRE RESULTS}

In 2011, the DQWG undertook a study into the Mariners' perception of data quality. The principle aim of this project was to produce a specification to be used in the development of any new means of representing data quality in future ENCs. This specification would take into account why mariners need data quality information, how mariners currently use data quality information, what mariners need from data quality information and the limitations of providing data quality information.

The analysis of the questionnaire results was based upon 574 responses, with $74 \%$ (421 respondents) of the sample population having over 10 years navigational experience and $63 \%$ (357 respondents) having in excess of 15 years navigational experience. In addition the results showed that a broad range of shipping sectors were represented. 
The questionnaire itself was of mixed methods design, incorporating qualitative questions into a dominantly quantitative structure (QUANqual). The questionnaire was split into three sections; Demographics, Mariners' perception of current methods of representing the quality of data in nautical charts and Future developments.

The key results from the study are reflected in the following specification [Harper 2012]:

- As a minimum the constituent elements of S-57 CATZOC (positional uncertainty, sounding uncertainty, features detected and seafloor coverage) must be encoded in S-101 ENCs for depth areas, as separate attributes

- All encoded data quality information must be discoverable

- Temporal degradation of data should be encoded

- New representation methods should be able to accommodate inputs such as dynamic tides, under keel allowance and vessel specific parameters. It is understood that international efforts on standardisation of display and mariner training address possible issues with user inputs.

- Where possible ENC attribute names should be more descriptive (eliminate 6 letter acronyms and make use of camelCase)

- Visualisation should take advantage of the mariner's preference for an on demand colour overlay

- Recommend to add ability for mariners to add notes to specific features, that again changes presentation of the feature (as an addition to the mariners' objects)

- Any representation method should be accompanied by an appropriate education strategy

\section{DRAFT S-101 DATA QUALITY UML MODEL}

The DQWG have been tasked by the IHO Transfer Standard Maintenance and Application Development working group (TSMAD), to develop a model for S-101 ENC data quality representation. Taking into account the results of the mariners' questionnaire, the DQWG have decided to focus on splitting the principle S-57 primary data quality indicator, CATZOC, into its constituent elements. The rational for this is that it will allow for a more flexible approach to capturing data quality, and reduce the likelihood that important data quality information is 'hidden' due to its inclusion in a composite indicator. In addition, it is an aspiration of the DQWG to include external inputs, as well as charted data into the future S-101 portrayal of data quality.

The Draft S-101 Data Quality UML model (Annex A) includes three S-100 data quality meta-features; QualityOfBathymetricData, QualityOfSurvey and QualityOfNonbathymetricData. Out of the three, only QualityOfBathymetricData is mandatory, and it carries under it all of the constituent elements of CATZOC, as well as the new attribute, QualityOfTemporalVariation. The inclusion of this new attribute was driven by the need to be able to represent the quality of charted data in terms of its currency. This is important as it is impossible to adequately represent the uncertainty of data caused by regular geomorphological processes such as sandwave migration, or extreme events such as earthquakes in an S-57 ENC.

\section{DRAFT ARCHITECTURE FOR S-101 DATA QUALITY REPRESENTATION}

One of the principle outcomes of the mariners' questionnaire was that a large number of chart users wanted to be able to utilise individual data quality indicators rather than rely upon a general indication of the quality of data in a particular area. However, this was not the case with all respondents. As a consequence the DQWG intend to develop a mechanism by which individually populated data quality indicators feed into an algorithm that in turn drives a composite indication of general data quality.

An example of such an approach is that suggested by Devillers et al. [2002], illustrated in figure 1. This approach, termed the "Multidimensional User Manual", works on a hierarchical traffic light system, which allows the user to drill down through the layers to find out what indicators have 
influenced the composite indicator. The rules governing how each indicator is coloured are held as either user controllable or predetermined settings.

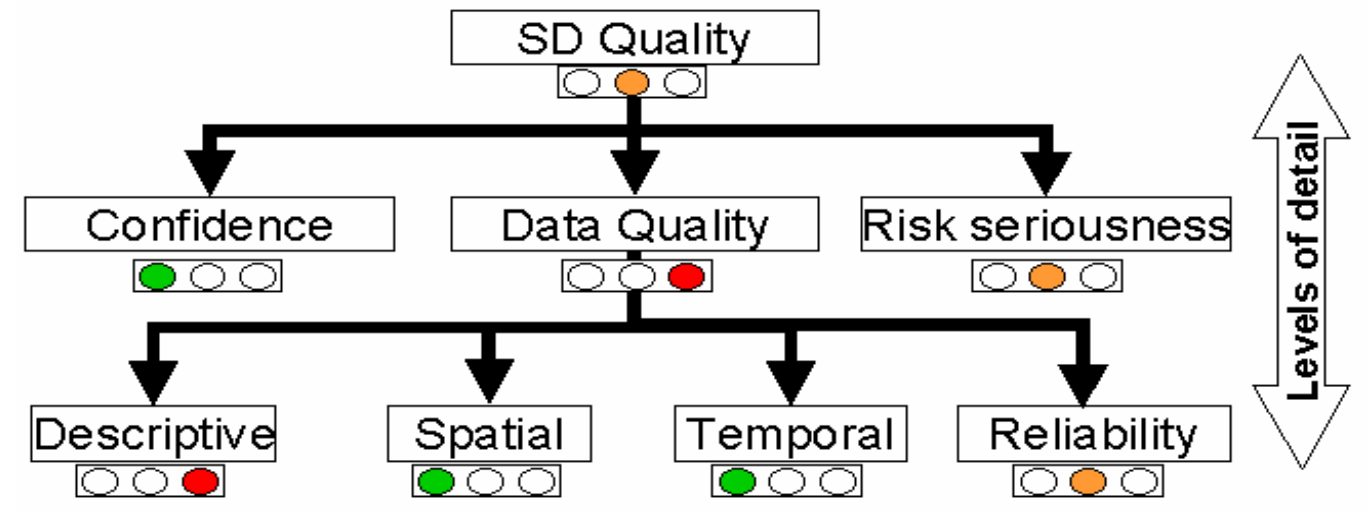

Figure 1. Example of Spatial Data Quality Indicators hierarchy [Devillers et al. 2002]

Figure 2 shows the DQWG's first draft of the high level architecture for S-101 ENC data quality portrayal. This approach builds upon the Multidimensional User Manual principles whilst allowing for the optional input of external models or parameters such as dynamic tides and vessel draught.

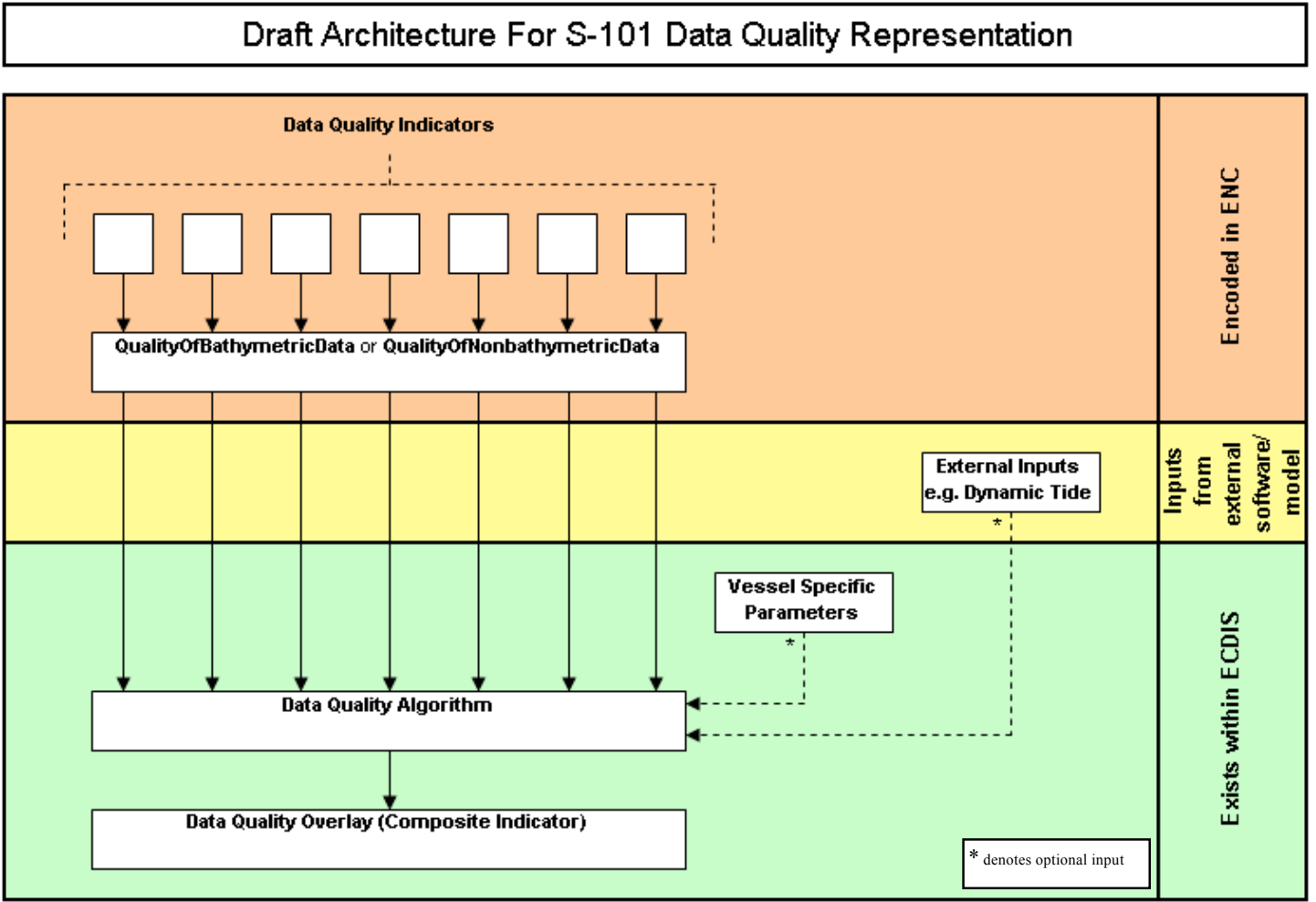

Figure 2. Draft Architecture for S-101 Data Quality Representation 


\section{USM STUDY INTO CHART QUALITY PORTRAYAL}

The DQWG have invited USM to use the results of the mariners' questionnaire and draft S-101 data quality UML model to research and develop the optimum method of representing data quality to chart users. Following initial meetings between DQWG members and USM staff and students, a two stage research project has been agreed.

Stage one will focus on developing a quality indicator visualisation test-bed that can be used to get feedback from mariners. This test bed will take the form of an S-57 Marine Information Object (MIO) that utilises the quality attributes that already exist in S-57 (POSACC, SOUACC and TECSOU), as well as a new quality attribute, CategoryOfTemporalVariation.

Stage two will build upon this experience and focus on developing more sophisticated methods of representing data quality. This will include research into composite indicators that are potentially capable of interacting with external inputs from models such as dynamic draught or dynamic tides, as well as the option to tailor visualisation depending on vessel specific parameters.

\section{Perception of the Problem}

USM have made the following initial observations regarding the nature and scope of the research problem:

1. The purpose of nautical charts and ancillary information is to facilitate informed decisionmaking by mariners and other chart users, without making the decisions for them.

2. Portrayal of chart quality indicators are most important during voyage planning, less important during voyage monitoring, but may also be important during emergencies. Emergency use may raise appropriate colour table encoding.

3. While this project may result in an array of parameters, options, features and components that may aid in representing data quality, these should be transparent to mariners and other end users, who must also be provided with the freedom to choose among these parameters, options, features and components according to their perceived priority, hence realizing the user's definition of safe navigation.

4. Component quality indicators, whose meaning is transparent to end-users, effectively facilitate informed decision-making.

5. The three quality components identified in Dorst \& Howlett [2012] (measurement uncertainty; completeness; currency) represent an appropriate starting point in defining indicators that are useful, intuitive and 'mariner-friendly'.

6. The assumption built into the above statement must be tested by eliciting feedback from mariners on the use of these quality components, sooner rather than later.

7. Since elements of these three quality components are already captured in the S-57 attributes POSACC, SOUACC, TECSOU, and SUREND, there is an opportunity to continue a dialogue with mariners about quality components immediately, using S-57 compliant ENCs.

8. In general, composite indicators on their own, such as CATZOC, or a replacement for CATZOC, risk incorporating a priori decision-making, which is inappropriate, and has an opaque meaning to end users. It may be that mariners will find a composite indicator useful, when accompanied by its component indicators. But this should be tested.

9. Past efforts to represent chart quality, whether by source diagrams or CATZOC encodings, represent chart quality in ways that may be useful to a hydrographer, but as indicated by the DQWG survey results, do not address the needs of, nor are easily interpreted by, a mariner. 
10. Efforts to develop a composite indicator to represent chart quality should proceed gradually, with mariner feedback at each step.

11. Regarding the visualisation of chart quality indicators, mariners have uniformly voiced approval and understanding of a scheme that encodes quality values into a green-yellow-red semi-transparent aerial overlay [Harper 2012]. We suggest a fourth colour, grey, for areas where quality indicator values are not available. We propose this as a starting point, while we develop and test more sophisticated ways of visualizing quality indicators.

These observations have been discussed with members of the DQWG and it has been agreed that they will form a strong foundation on which to progress this research. The results from the first stage of the project will be presented to the DQWG at the next meeting in July 2013.

\section{CONCLUSION}

The DQWG study of mariners' perception of data quality contributes to a significant change in the approach of the international chart producing community in deciding what information is presented on a chart. The DQWG is committed to developing data quality representation methods that are driven by the needs of the mariner. In order to fully realise this aim, the DQWG is interacting with academic institutions and industry experts in an attempt to harness and strengthen the collective wisdom of the group. It is hoped that mariners will recognise their input in the resulting solution. 


\section{REFERENCES}

Devillers, Gervais, Bedard, Jeansoulin [2002] Spatial data quality: from metadata to quality indicators and contextual end-user manual, OEEPE/ISPRS Joint Workshop on Spatial Data Quality Management, pp45-55, March 21-22, Istanbul

Dorst \& Howlett [2012] Safe Navigation with uncertain hydrographic data. Hydro International, June 2012, pp18-21.

Harper [2012] A Specification For Developing a New Method of Visualising Data Quality in Electronic Navigational Charts. Canadian Hydrographic Conference 2012, May 15-17, Niagara Falls

\section{BIOGRAPHIES}

Sam HARPER works as a Senior Bathymetric Appraisal Officer for the United Kingdom Hydrographic Office Seabed Data Centre and is a graduate of Plymouth University's MSc Hydrography programme. $\mathrm{He}$ is also the UKHO representative on the IHO Data Quality Working Group, a Director of the Hydrographic Society UK and the Hon. Secretary of the Hydrographic Society UK South West Region. Prior to specializing in hydrography, Sam worked in GIS and marine science.

David WELLS currently teaches courses in Applied Bathymetry and Kinematic Positioning at the University of Southern Mississippi; is one of four instructors who have taught nearly 60 short courses on the use of multibeam sonar for marine surveys; and is a Professor Emeritus at the University of New Brunswick, where he taught courses on hydrographic topics for 18 years, and established the UNB Ocean Mapping Group. Determining and representing hydrographic uncertainty is one of his main interests.

Kandice GUNNING is a graduate of the Geomatics Engineering and Land Management Department at the University of the West Indies, St.Augustine. She is currently a hydrography student and teaching assistant at the Department of Marine Science at the University of Southern Mississippi.

\section{CONTACT DETAILS}

Sam HARPER

United Kingdom Hydrographic Office

Admiralty Way

Taunton

United Kingdom

Tel.: +44 (0) 1823337900 Ext. 3843

Fax: +44 (0) 1823284077

Email: samuel.harper@ukho.gov.uk 
Web site: www.ukho.gov.uk 


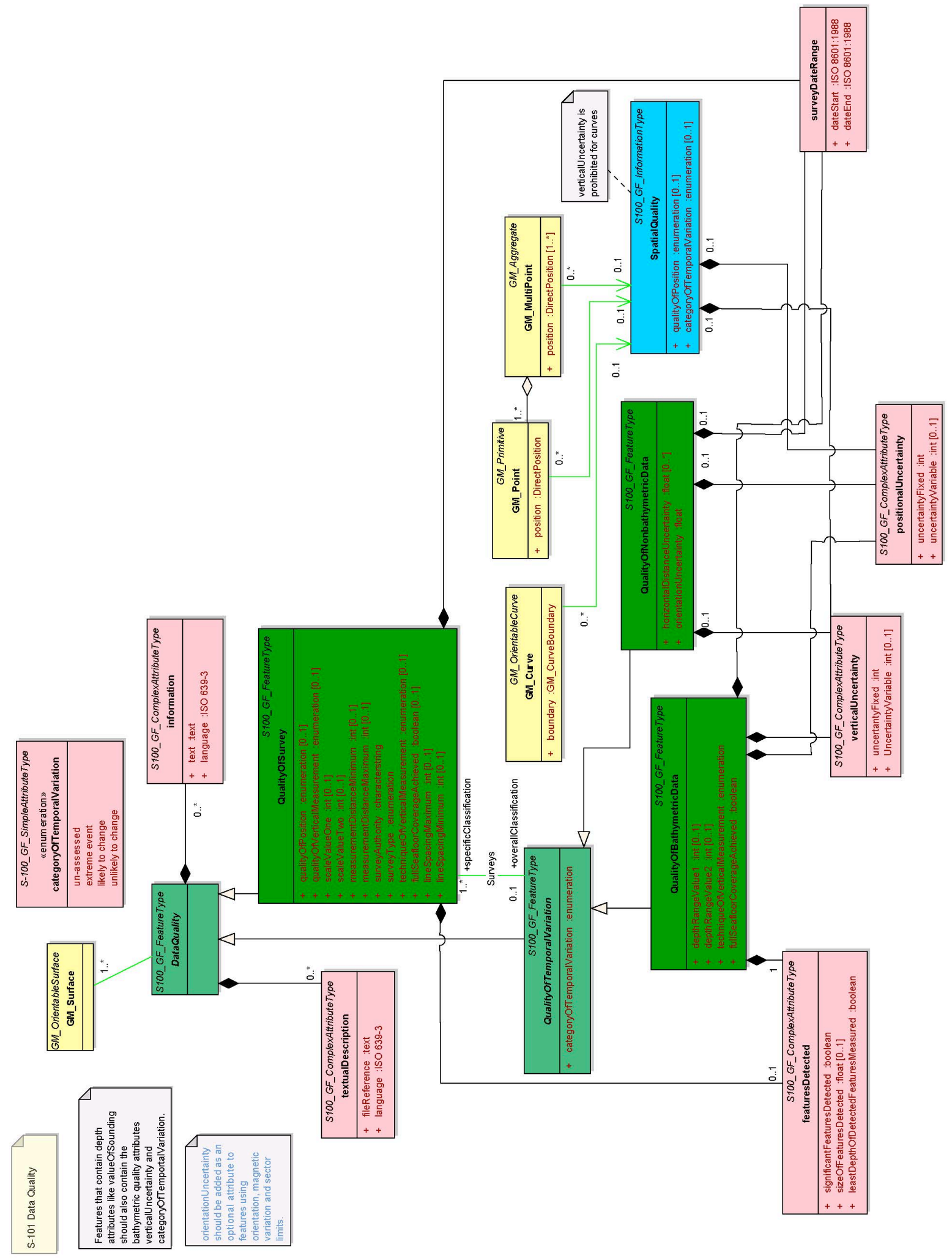

